\title{
Editorial
}

Hautarzt 2013 - 64:893-893

DOI 10.1007/s00105-013-2606-1

Online publiziert: 29. November 2013

(c) Springer-Verlag Berlin Heidelberg 2013
R.U. Peter ${ }^{1}$ J. Krutmann ${ }^{2}$

${ }^{1}$ Gefäß- und Hautzentrum, Medizinisches Versorgungszentrum, Blaustein

${ }^{2}$ IUF - Leibniz-Institut für umweltmedizinische Forschung g GmbH, IUF Leibniz Research Institute for Environmental Medicine, Düsseldorf

\section{Strahlenerkrankungen der Haut}

Sehr geehrte Leserinnen und Leser,

vor Ihnen liegt ein Leitthemenheft, das sich mit einer den meisten Hautärzten wenig präsenten und vielleicht auch gelegentlich verdrängten Problematik beschäftigt: den klinischen Folgen und therapeutischen sowie präventiven Optionen einer Überexposition der Haut mit ionisierenden Strahlen.

Auch wenn es sich hierbei um seltene Schadensereignisse handelt, sind Schlagwörter wie Nuklearkatastrophe, Nuklearterrorismus usw. nicht erst seit Fukushima wieder von allgemeinem Interesse.

Die meisten heute tätigen Dermatologen verfügen über wenig oder keine eigenen praktischen Erfahrungen in der Röntgentherapie, somit sind ihnen die klinischen Folgen einer Exposition der Haut mit ionisierenden Strahlen meist nicht mehr aus eigener Anschauung bekannt.

\section{》) Die meisten Dermatologen haben keine Erfahrungen mit Strahlenerkrankungen der Haut}

Die vorliegenden Übersichtsarbeiten sollen einerseits Ihnen als in Klinik oder Praxis tätigen Dermatologen Anhaltspunkte zur Erkennung, Prävention und Therapie geben bzw. grundsätzlich für diese Schadensszenarien sensibilisieren, die im Einzelfall sehr viel weniger spektakulär daherkommen können als im Rahmen einer großen Katastrophe.

Zum anderen sollen neue Erkenntnisse hinsichtlich des Erythemzeitpunkts und der -intensität als prognostisch relevantem Faktor und das Potenzial der Stamm- zelltherapie als neue mögliche Therapieoption aufgezeigt werden.

Vielen mögen diese Befunde und die aufgezeigten Therapieoptionen weit entfernt erscheinen, aber sie können schnell Realität werden. Dann bedarf es vor allem fach- und sachkundiger Dermatologen, denn bei allen denkbaren Unfallszenarien ist das Hautorgan vorrangig involviert, und die betroffenen Patienten benötigen einerseits rasche und professionelle Hilfe, andererseits jahre- und jahrzehntelange Betreuung und Nachsorge.

Wir wünschen Ihnen viel Spaß bei der Lektüre und stehen für Nachfragen gerne zur Verfügung.

Die Heftherausgeber

Prof. Dr. Dr. Ralf U. Peter

Prof. Dr. Jean Krutmann

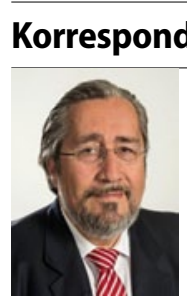

Gefäß- und Hautzentrum, Medizinisches

Versorgungszentrum Erhard-Grözinger-Str. 102, 89134 Blaustein peter@blausteinpraxis.de

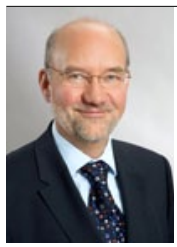

Univ.-Prof. Dr. J. Krutmann IUF - Leibniz-Institut für umweltmedizinische Forschung $\mathrm{gGmbH}$, IUF - Leibniz Research Institute for Environmental Medicine Auf'm Hennekamp 50, 40225 Düsseldorf Jean.Krutmann@ IUF-Duesseldorf.de

Interessenkonflikt. R.U. Peter und J. Krutmann geben an, dass kein Interessenkonflikt besteht. 